\title{
Methods in Enzymology, Vol. 480, Glycobiology
}

\author{
(M. Fukuda (ed.), Elsevier, Amsterdam-Boston-Heidelberg-London-New-York-Oxford-Paris, \\ 2010, 581 p., \$182)
}

DOI: $10.1134 / \mathrm{S} 0006297911100142$

This book consists of five sections including 25 chapters written by an international group of authors. The volume covers proteoglycan function, infection, immunity, carbohydrate-binding proteins including galectin, and new developments including O-glycosylation in Notch and related signaling.

Section I (chapters 1-4) highlights proteoglycans and sulfotransferases. There are data and discussions related to proteoglycan signaling networks, roles of Drosophila glypican, various assays for measuring sulfatase activity, and analysis of heparan sulfate structure and biological activity.

Section II (chapters 5-13) is devoted to lectins, immunity, and infection. Special chapters of this section include microbe-associated molecular patterns in innate immunity, structural and functional analysis of glycosphingolipids of Schistosoma mansoni, analysis of biotoxicity of body lectins and other cytoplasmic proteins, lectins of endoplasmic reticulum with homology with mannose-6phosphate receptors, and various aspects of glycobiology related to galectins.

Section III (chapters 14 and 15) is devoted to Drosophila. It contains data about glycan spectrum and characterization of glycosyltransferases and transporters that contribute to proteoglycan synthesis.

Section IV (chapters 16 and 17) characterizes Notch signaling. The modification of the extracellular domain of notch receptors and regulation of Notch signaling via $\mathrm{O}-$ glycosylation in Drosophila are discussed.

Section V (chapters 18-25) summarizes new comprehensive data in development. There are discussions about O-fucosylation of thrombospondin, specificity of glycan-binding proteins, functional roles of bisecting GlcNAc in integrin-mediated cell adhesion, and other topics related to glycobiology of development.

The book contains author and subject indexes, a bibliography for each chapter, and also color photographs placed at the end of the book.

Like the previous Vols. 478 and 479 , the book is very informative, and it is novel both in terms of problems discussed and also new methods used for glycobiology.

This book will be very useful to a wide variety of readers from graduate students, researchers in academia, in industry, and to those who teach glycobiology and glycosciences at various levels. 